\title{
Neosporose bovina: avaliação da transmissão vertical e fração atribuível de aborto em uma população de bovinos no Estado do Rio Grande do Sul ${ }^{1}$
}

\author{
Héber E. Hein ${ }^{2}$, Gustavo Machado ${ }^{2}$, Igor C.S. Miranda², Eduardo F. Costa², \\ Débora C.P. Pellegrini² ${ }^{2}$ David Driemeier ${ }^{3}$ e Luís G. Corbellini ${ }^{2 *}$
}

\begin{abstract}
Hein H.E., Machado G., Miranda I.C.S., Costa E.F., Pellegrini D.C.P., Driemeier D. \& Corbellini L.G. 2012. [Bovine neosporosis: evaluation of vertical transmission and population etiological fraction of abortion in a bovine population in the State of Rio Grande do Sul, Brazil]. Neosporose bovina: avaliação da transmissão vertical e fração atribuível de aborto em uma população de bovinos no Estado do Rio Grande do Sul. Pesquisa Veterinária Brasileira 32(5):396-400. Laboratório de Epidemiologia Veterinária, Setor de Medicina Veterinária Preventiva, Faculdade de Veterinária, Universidade Federal do Rio Grande do Sul, Avenida Bento Gonçalves 9090, Porto Alegre, RS 91540-000, Brazil. E-mail: luis.corbellini@ufrgs.br

The protozoa Nesporora caninum is a parasite that causes great economic and reproductive losses in cattle worldwide. The objective of this study was to verify the association between abortion and the presence of antibodies against $N$. caninum and the vertical transmission as a means of maintaining the infection in cattle herds in Rio Grande do Sul by matching the serology of mothers and daughters. Sampling was performed in 60 dairy farms of two regions of the state, where blood was collected from $40 \%$ of the herds for the detection of anti- $N$. caninum by indirect immunofluorescence (IFI). To verify the association between abortion and seropositivity we used the univariate logistic regression test, and for the serology of mothers and daughters the chi-square McNemar test for paired data. In comparing serology and abortion data, a prevalence of 58.5\% (24/41) among cattle with history of abortion, and $16.4 \%(199 / 1215)$ prevalence between those with no history of abortion was found; seropositive animals were 7.21 times more likely $(95 \% \mathrm{CI}, 3.65-14.32)$ to have previously aborted (Wald statistic $\chi^{2}=44.93, P<0.001$ ). The fraction affected by neosporosis in the population studied was estimated as $9.73 \%\left(\lambda_{\text {pop }}\right)$. The serologic result of each mother was paired with her daughter and showed by the McNemar chi-square $\left(\chi^{2}=59.84, P<0.001\right)$ significant association between the serology of mothers and daughters, suggesting vertical transmission. It is worth to note the importance of serological monitoring for $N$. caninu to avoid maintenance of animals that may serve as carries of the parasite reservoir on the farms.
\end{abstract}

INDEX TERMS: Neospora caninum, abortion, vertical transmission, etiological fraction.

RESUMO.- O protozoário Nespora caninum é um parasito que causa grandes perdas reprodutivas e econômicas em bovinos no mundo inteiro. Os objetivos deste estudo foram

\footnotetext{
${ }^{1}$ Recebido em 1 de outubro de 2011.

Aceito para publicação em 20 de dezembro de 2011.

${ }^{2}$ Laboratório de Epidemiologia Veterinária (Epilab), Setor de Medicina Veterinária Preventiva, Faculdade de Veterinária (Favet), Universidade Federal do Rio Grande do Sul (UFRGS), Av. Bento Gonçalves 9090, Porto Alegre, RS 91540-000, Brasil. *Autor para correspondência: luis.corbellini@ufrgs.br

${ }^{3}$ Setor de Patologia Veterinária, Favet-UFRGS, Porto Alegre, RS.
}

verificar tanto a associação entre o histórico de aborto e a presença de anticorpos contra $N$. caninum, quanto a transmissão vertical como forma de manutenção da infecção nos rebanhos bovinos em regiões do Rio Grande do Sul, através da sorologia pareada de mães e filhas. Foi realizada amostragem de 60 propriedades distribuídas em duas regiões do Rio Grande do Sul, das quais foi coletado sangue de $40 \%$ dos animais presentes para a detecção de anticorpos anti- N. caninum por imunofluorescência indireta (IFI). Para verificar a relação aborto e soropositividade foi utilizado o teste de regressão logística univariada, e para sorologia 
de mães e filhas o teste de qui-quadrado de McNemar para dados pareados. Foram confrontados os dados de sorologia e aborto, sendo encontrada a frequência de 58,5\% (24/41) de soropositivos quando havia histórico de aborto, e 16,4\% (199/1215) dentre os sem histórico de aborto. Os animais soropositivos apresentaram um risco 7,21 (IC 95\%, 3,65$14,32)$ vezes maior de possuir histórico de abortamento (estatística de Wald $\chi^{2}=44,93, P<0,001$ ). A fração atribuível à neosporose como causa de aborto na população em risco nas duas regiões foi estimada em $9,73 \%\left(\lambda_{\text {pop }}\right)$. 0 resultado sorológico de cada mãe foi pareado com o de sua filha e, pelo teste de qui-quadrado de McNemar $\left(\chi^{2}=59,84\right.$, $P<0,001)$, houve associação significativa entre as sorologias de mães e filhas, sugerindo transmissão vertical. Ressalta-se ainda a importância do acompanhamento sorológico para $N$. caninum, evitando assim manutenção de animais portadores que sirvam como reservatório do protozoário nas propriedades.

TERMOS DE INDEXAÇÃO: Neospora caninum, aborto, transmissão vertical, fração atribuível.

\section{INTRODUÇÃO}

O protozoário Neospora caninum foi descrito primeiramente em cães por Dubey et al. (1988), causando principalmente uma doença neuromuscular (Dubey \& Lindsay 1996). Este parasito tem sido também associado como uma das principais causas de aborto bovino (Dubey 1999), podendo ainda interferir na produção leite e na produtividade do rebanho (Thurmond \& Hietala 1996). Inúmeras espécies de animais selvagens e domésticas têm sido identificadas como hospedeiros intermediários do $N$. caninum (Dubey \& Lindsay 1996), porém foi estabelecido que bovinos sejam os principais, uma vez que animais infectados por $N$. caninum permanecem carreadores do parasito durante toda vida e não há sinais claros de proteção imunológica desenvolvida pelo hospedeiro (Innes et al. 2002). A infecção horizontal ocorre devido a presença do principal hospedeiro definitivo, o cão (McAllister et al. 1998, Gondim et al. 2004), que elimina oocistos do parasito através das fezes e que podem contaminar utensílios e alimentos utilizados no manejo do rebanho e alimentação dos bovinos que, uma vez infectados, podem manter a infecção entre as gerações através da transmissão vertical (Dubey et al. 2007). A infecção tem como indicador a presença de anticorpos específicos anti- $N$. caninum tanto no sangue quanto no leite (Björkman et al. 1996). Estudos de prevalência de neosporose têm sido desenvolvidos em praticamente todos os continentes, sendo as prevalências altas na maioria dos estudos, o que remete à importância da doença em relação ao aborto e consequentemente às perdas econômicas relacionadas a este fato. Há ainda variações de prevalência entre 0 e 91,2\%, podendo ser explicadas pela influência de fatores inerentes à variação climática entre países e regiões, por exemplo (Dubey et al. 2007). Em bovinos leiteiros a transmissão ocorre principalmente pela via vertical, o que possibilitaria a manutenção da infecção nos rebanhos por diversas gerações (Schares et al. 1998). Porém, fêmeas infectadas e testadas positivamente para $N$. canimum pode- rão gerar bezerros soronegativos que posteriormente podem vir a se infectar através da transmissão horizontal pela ingestão de oocistos (McAllister et al. 1998). A transmissão vertical já foi determinada entre soros pareados de mães e filhas em diversos países (Schares et al. 1998, Davison et al. 1999, Stenlund et al. 1999).

Inúmeros estudos de transmissão vertical e horizontal de neosporose já foram realizados, porém não há informação adequada para análise do caráter da transmissão vertical no Brasil bem como a fração atribuível de aborto causado por N. caninum numa determinada população. Com o auxílio de programas específicos, dados relativos ao diagnóstico de neosporose disponibilizados pelo Laboratório de Epidemiologia da Faculdade de Veterinária da Universidade Federal do Rio Grande do Sul (Epilab-Favet/UFRGS) foram utilizados para as análises estatísticas. Os objetivos do estudo foram verificar tanto a associação entre histórico de aborto e a presença de anticorpos contra $N$. caninum e a fração atribuível, quanto a transmissão vertical como forma de manutenção da infecção nos rebanhos bovinos em regiões do Rio Grande do Sul, através da sorologia pareada de mães e filhas.

\section{MATERIAL E MÉTODOS}

Os dados para análise foram obtidos de um estudo transversal de neosporose bovina em duas regiões do Rio Grande do Sul (Corbellini et al. 2006), no qual foram amostradas de forma aleatória simples 30 propriedades de cada uma das regiões estudadas, localizadas no noroeste e sudeste do estado, a partir de um total de 800 e 150 propriedades de duas cooperativas, respectivamente. Um total de 1463 animais foi amostrado a partir de um efetivo de 3872 bovinos das 60 propriedades. Após o sorteio dos animais na propriedade, perguntou-se ao proprietário sobre o histórico de aborto dos animais amostrados. Contudo no presente estudo utilizaram-se dados de 1256 animais amostrados, pois fêmeas com idade inferior a 15 meses foram retiradas, uma vez que estes animais têm uma probabilidade muito baixa de possuir histórico de aborto. 0 teste de imunofluorescência indireta (IFI) foi realizado para detecção de anticorpos contra $N$. caninum nas amostras de soro dos animais. 0 ponto de corte considerado indicativo de infecção foi de 1:200.

A cooperativa da região noroeste continha os registros computacionais completos dos animais de cada propriedade, bem como a genealogia. Desta forma, dentre os animais amostrados desta população, 101 pares de mães e filhas puderam ser selecionados da amostra para a avaliação da transmissão vertical do protozoário através da detecção de anticorpos nos pares de mães-filhas.

\section{Análise estatística}

Foi utilizado o modelo de regressão logística univariado com o objetivo de verificar a hipótese de associação entre a variável dependente Y (histórico de aborto) e a variável independente $\mathrm{X}$ (sorologia para $N$. caninum) e estatística de Wald para verificar a significância da associação. 0 modelo foi testado para verificação de ajustamento pelo teste de Hosmer \& Lemeshow (1980). A idade dos animais foi utilizada para ajuste do estimador do Odds, visto ser um possível confundidor na associação entre histórico de aborto e sorologia para $N$. caninum, pois animais mais velhos possuem maior risco de abortamento pelo fato de terem tido um maior número de gestações. Para isso, mudanças nos parâmetros dos coeficientes $>25 \%$ foram avaliados entre os dois modelos 
(com e sem a variável independente idade). A fração de risco atribuível $\left(\lambda_{\text {pop }}\right)$ às causas de aborto relativos à infecção por N. caninum foi estimada através da seguinte equação $\lambda_{\text {pop }}=1-[\{c(b+d)\} /$ $\{\mathrm{d}(\mathrm{a}+\mathrm{c})\}]$ (Thrusfield 2007), onde $a$ e $d$ (concordantes), $b$ e $c$ (discordantes) correspondem às células de uma tabela de contingência $2 \times 2$. Para a análise dos resultados sorológicos de mães e filhas foi utilizado o teste de qui-quadrado de McNemar para dados pareados. Um nível de significância $(\alpha)$ de $5 \%$ foi utilizado. As análises foram realizadas nos programas MS Excel 2010, EpiInfo 2000 e SAS versão 9.1 (SAS Institute).

\section{RESULTADOS E DISCUSSÃO}

Os dados de sorologia e histórico de aborto foram cruzados, a frequência encontrada foi de 58,5\% (24/41) de soropositivos quando havia histórico de aborto, e $16,4 \%$ (199/1215) dentre os sem histórico de aborto (Quadro 1). Na Argentina, animais soropositivos tiveram $85 \%$ mais chances de abortar que animais soronegativos, o que sugere maior risco a esta população (Moore et al. 2009). A possível associação existente entre aborto e sorologia para Neospora caninum foi testada através da regressão logística e animais soropositivos apresentaram um risco 7,21 (IC $95 \%, 3,65-14,32$ ) vezes maior de possuir histórico de abortamento (Estatística de Wald $=39,33 P<0,001$ ), fato que demonstra uma forte associação entre fêmeas com histórico de aborto e positividade, sugerindo que a infecção por $N$. caninum pode ser uma importante causa de aborto. A adequação do modelo foi confirmada pelo teste de Hosmer \& Lemeshow $(P=0.53)$. Estudos sorológicos observaram que fêmeas positivas para $N$. caninum tiveram de 2 a 7,4 vezes mais chances de terem histórico de aborto (Paré et al. 1997, Thurmond \& Hietala 1997b, Corbellini et al. 2002, Pfeiffer et al. 2002).

Quadro 1. Distribuição entre sorologias para Neospora caninum e histórico de aborto em animais maiores que $\mathbf{1 5}$ meses de idade de 60 propriedades leiteiras do Rio Grande do Sul

\begin{tabular}{lccc}
\hline \multirow{2}{*}{$\begin{array}{c}\text { Sorologia Neospora } \\
\text { caninum }\end{array}$} & \multicolumn{3}{c}{ Histórico de aborto* } \\
\cline { 2 - 4 } & Sim (\%) & Não (\%) & Total \\
\hline Positivo (\%) & $24(58,5)$ & $199(16,4)$ & 223 \\
Negativo & 17 & 1016 & 1033 \\
Total & 41 & 1215 & 1256 \\
* Os indicadores demonstrados são os de prevalência de \\
\multicolumn{3}{l}{$\begin{array}{l}\text { Neospora caninum nos cortes de animais com e sem his- } \\
\text { tórico de aborto. }\end{array}$}
\end{tabular}

Não há dados absolutos do prejuízo causado pela neosporose devido às dificuldades em documentar os casos, visto o aborto ser o único sinal aparente da doença (Dubey \& Schares 2011), no entanto no presente estudo foi estimada indiretamente a fração de risco atribuível à neosporose como causa de aborto, ou seja, esta estimativa demonstra a proporção da doença na população, cuja enfermidade possa ser atribuída à exposição. Neste caso, foi estimado que $N$. caninum foi responsável por $9,73 \%\left(\lambda_{\text {pop }}\right)$ dos abortos na população em risco nas duas regiões, o que concorda com Davison et al. (1999), que relataram que $12,5 \%$ dos abortos em gado leiteiro na Inglaterra e País de Gales são atribuídos à infecção por $N$. caninum. Estes problemas reprodutivos podem ocasionar importantes perdas econômicas, como relatado na Suíça, onde os custos relacionados à infecção variaram entre 81 e 1875 euros por propriedade positiva (Häsler et al. 2008). Além das perdas reprodutivas diretas, relacionadas ao aborto, custos com atendimento veterinário, diagnóstico, substituição e descarte de animais, maior intervalo entre partos e retorno para cobertura (Dubey \& Schares 2011), possível diminuição na produção, qualidade e composição do leite (Thurmond et al. 1997a, Trees et al. 1999, Tiwari et al. 2007), e ainda baixo ganho de peso em bovinos de corte (Hoar et al. 2007, Moré et al. 2010) também podem ocorrer.

Do mesmo estudo (Corbellini et al. 2006) foram obtidos dados referentes à sorologia de 101 vacas e também de suas filhas. A frequência de soropositivos encontrada nos dois estratos (mães e filhas) foi semelhante, sendo de $12,9 \%$ (13/101) nas mães, e de 11,9\% (12/101) nas filhas (Quadro 2); percebe-se a frequência maior de filhas soropositivas entre as mães também soropositivas, $69,2 \%$ (9/13), se comparado com mães soronegativas que apresentaram apenas 3,4\% (3/88) de filhas soropositivas (Quadro 2). Parte destes bezerros descendentes de mães positivas podem ter sido testados negativamente no momento do estudo por falhas do teste, seja por identificar mães falso-positivas ou filhas falso-negativas, no entanto eventualmente as filhas negativas neste estudo podem entrar em contato com oocistos, seja no ambiente, fonte de água ou alimento, e possivelmente se tornar infectadas, assim como Frössling et al. (2005) que testaram 1321 amostras e encontraram um número reduzido de bezerros testados positivos porém suas mães negativas.

Quadro 2. Distribuição pareada entre sorologias para Neospora caninum de mães e suas filhas por estrato

\begin{tabular}{lcccc}
\hline \multirow{2}{*}{ Mães } & \multicolumn{2}{c}{ Filhas } & Total & $\begin{array}{c}\text { \% de filhas } \\
\text { Soropositivas* }\end{array}$ \\
\cline { 2 - 3 } & Positivas & Negativas & & 69,2 \\
\hline Positivas & 9 & 4 & 13 & 3,4 \\
Negativas & 3 & 85 & 88 & 11,9 \\
\hline Total & 12 & 89 & $\mathbf{1 0 1}$ & \\
\hline
\end{tabular}

*Frequência de filhas soropositivas nos estratos de mães soropositivas e mães soronegativas.

O resultado sorológico de cada mãe foi pareado com o de sua filha e, pelo teste de qui-quadrado de McNemar para pares concordantes $\left(\chi^{2}=59,84, P<0,001\right)$ houve associação significativa entre as sorologias de mães e filhas, sustentando a hipótese de que a transmissão vertical é uma importante via de manutenção da neosporose dentro de rebanhos leiteiros por sucessivas gerações (Anderson et al. 1997), tanto no Brasil como em outros países (Dubey et al. 2007), podendo os títulos de anticorpos de filhas ser até 20 vezes maior que o de suas mães (Moré et al. 2009). Com frequências variando entre 37,1\% (Moré et al. 2009) e $95,2 \%$ (Davison et al. 1999) de transmissão vertical, verificou-se no presente estudo, taxa de 69,2\% (9/13), semelhante a Romero \& Frankena (2003) em estudo na Costa Rica $(63,7 \%)$, frequência esta considerada alta, o que confirma a importância desta como principal via de transmissão e causa de prejuízos na produção em bovinocultura de leite e carne. 
A transmissão vertical de mãe para filha e ingestão pós-natal de oocisto são os únicos dois meios de transmissão comprovados, o N. caninum é considerado um dos parasitos mais eficientes em transmissão transplacentária entre todos os agentes infecciosos que acometem bovinos (Dubey \& Schares 2011). A transmissão transplacentária pode ocorrer por aquisição de infecção pós-natal através da ingestão de oocitos (exógena) ou por reativação de infecção em vacas cronicamente infectadas (endógena) e a taxa de transmissão pode diferir nestes dois cenários (Williams et al. 2009). Estudos sorológicos usando sangue pós colostral mostraram que 81 a $95 \%$ das vacas positivas transmitem o parasito para seus descendentes (Dijkstra et al. 2001), já na Argentina a taxa foi de $37,1 \%$ e mães com títulos mais altos tiveram mais chances de infectar os filhos que mães com títulos mais baixos (Moré et al. 2009).

\section{CONCLUSÕES}

A associação entre histórico de aborto e sorologia para Nesopora caninum é uma situação que ocorre de forma relevante e frequente, visto que os animais soropositivos apresentaram um risco 7,21 (IC 95\%, 3,65-14,32) vezes maior de possuir histórico de abortamento. Isto demonstra que alternativas de controle devem ser adotadas para o controle da infecção dentro dos rebanhos bovinos, seja pela eliminação de animais soropositivos, aquisição de animais negativos, ou ainda pela correta destinação de produtos de aborto e controle do acesso de cães e outros animais às criações, evitando possíveis perdas econômicas.

A verificação de uma taxa de transmissão vertical de 69,2\% na região noroeste do Rio Grande do Sul sugere que esta pode ser uma das principais rotas de transmissão e manutenção da neosporose entre gerações nos sistemas de criação bovina. Visto que os bezerros podem nascer infectados, porém assintomáticos, é importante seu acompanhamento sorológico, evitando assim a presença de animais portadores que sirvam como reservatório do protozoário nas propriedades.

Agradecimentos.- Este trabalho foi financiado pelo Instituto Nacional de Ciência e Tecnologia (CNPq, Proc.570365/2008-2).

\section{REFERÊNCIAS}

Anderson M.L., Reynolds J.P., Rowe J.D., Sverlow K.W., Packham A.E., Baar B.C. \& Conrad P.A. 1997. Evidence of vertical transmission of Neospora sp. infection in dairy cattle. J. Am. Vet. Med. Assoc. 8:1169-1172.

Björkman C., Johansson O., Stenlund S., Holmdahl O.J. \& Uggla A. 1996. Neospora species infection in a herd of dairy cattle. J. Am. Vet. Med. Assoc. 208:1441-1444.

Corbellini L.G., Driemeier D., Cruz C.E.F., Gondim L.F.P. \& Wald V. 2002. Neosporosis as a cause of abortion in dairy cattle in Rio Grande do Sul. Vet. Parasitol. 103:195-202.

Corbellini L.G., Smith D.R., Pescador C.A., Schmitz M., Correa A., Steffen D.J. \& Driemeier D. 2006. Herd-level risk factors for Neospora caninum seroprevalence in dairy farms in southern Brazil. Prev. Vet. Med. 74:130-141.

Davison H.C., Otter A. \& Trees A.J. 1999. Estimation of vertical and horizontal transmission parameters of Neospora caninum infections in dairy cattle. Int. J. Parasitol. 29:1683-1689.

Dijkstra T., Barkema H.W., Eysker M. \& Wouda W. 2001. Evidence of post-natal transmission of Neospora caninum in Dutch dairy herds. Int. J. Parasitol. 31:209-215.
Dubey J.P. 1999. Recent advances in Neospora and neosporosis. Vet. Parasitol. 84:349- 367.

Dubey J.P., Carpenter J.L., Speer C.A., Topper M.J. \& Uggla A. 1988. A newly recognized fatal protozoan disease of dogs. J. Am. Vet. Med. Assoc. 192(9):1269-1285.

Dubey J.P. \& Lindsay D.S. 1996. A review of Neospora caninum and neosporosis. Vet. Parasitol. 67:1-59.

Dubey J.P. \& Schares G. 2011. Neosporosis in animals: The last five years. Vet. Parasitol. 180:90-108.

Dubey J.P., Schares G. \& Ortega-mora L.M. 2007. Epidemiology and control of Neosporosis and Neospora caninum. Clin. Microbiol. Rev. 20:323-367.

Frössling J., Uggla A. \& Björkman C. 2005. Prevalence and transmission of Neospora caninum within infected Swedish dairy herds. Vet. Parasitol. 128:209-218.

Gondim L.F., McAllister M.M., Pitt W.C. \& Zemlicka D.E. 2004. Coyotes (Canislatrans) are definitive hosts of Neospora caninum. Int. J. Parasitol. 34:159-161.

Häsler B., Stärk K., Gottstein B. \& Reist M. 2008. Epidemiologische und finanzielle Entscheidungsgrundlagen zur Kontrolle von Neospora caninum auf Schweizer Milchviehbetrieben. Schweiz. Arch. Tierheilkd. 150:273-280.

Hoar B.R., McQuarry A.C. \& Hietala S.K. 2007. Prevalence of Neospora caninum and persistent infection with bovine viral diarrhea virus in dairy-breed steers in a feedlot. J. Am. Vet. Med. Assoc. 230:1038-1043.

Hosmer D. \& Lemeshow S. 1980. Goodness-of-fit tests for the multiple logistic regression model. Commun. Stat. A, Theor. Methods 9:1043-1069.

Innes E.A., Andrianarivo A.G., Bjorkman C., Williams D.J. \& Conrad P.A. 2002. Immune responses to Neospora caninum and prospects for vaccination. Trends. Parasitol. 18:497-504.

McAllister M.M., Dubey J.P., Lindsay D.S., Jolley W.R., Wills R.A. \& Mcguire A.M. 1998. Dogs are definitive hosts of Neospora caninum. Int. J. Parasitol. 28:1473-1478.

Moore D.P., Pérez A., Agliano S., Brace M., Cantón G., Cano D., Leunda M.R., Odeón A.C., Odriozola E. \& Campero C.M. 2009. Risk factors associated with Neospora caninum infections in cattle in Argentina. Vet. Parasitol. 161:122-125.

Moré G., Bacigalupe D., Basso W., Rambeaud M., Beltrame F., Ramirez B., Venturini M.C. \& Venturini L. 2009. Frequency of horizontal and vertical transmission for Sarcocystis cruzi and Neospora caninum in dairy cattle. Vet. Parasitol. 160:51-54.

Moré G., Bacigalupe D., Basso W., Rambeaud M., Venturini M.C. \& Venturini L. 2010. Serologic profiles for Sarcocystis sp. and Neospora caninum and productive performance in naturally infected beef calves. Parasitol. Res. 106:689-693.

Paré J., Thurmond M.C. \& Hietala S.K. 1997. Neospora caninum antibodies in cows during pregnancy as a predictor of congenital infection and abortion. J. Parasitol. 83:82-87.

Pfeiffer D.U., Williamson N.B., Reichel M.P., Wichtel J.J. \& Teague W.R. 2002. A longitudinal study of Neospora caninum infection on a dairy farm in New Zealand. Prev. Vet. Med. 54:11-24.

Romero J.J. \& Frankena K. 2003. The effect of the dam-calf relationship on serostatus to Neospora caninum on 20 Costa Rican dairy farms. Vet. Parasitol. 114:159-171.

Schares G., Peters M., Wurm R., Barwald A. \& Conraths F.J. 1998. The efficiency of vertical transmission of Neospora caninum in dairy cattle analysed by serological techniques. Vet. Parasitol. 80:87-98.

Stenlund S., Kindahl H., Magnusson U., Uggla A. \& Björkman C. 1999. Serum antibody profile and reproductive performance during two consecutive pregnancies of cows naturally infected with Neospora caninum. Vet. Parasitol. 85:227-234.

Thurmond M.C. \& Hietala S.K. 1996. Culling associated with Neospora caninum infection in dairy cows. Am. J. Vet. Res. 57:1559-1562.

Thurmond M.C. \& Hietala S.K. 1997a. Effect of Neospora caninum infection on milk production in first-lactation dairy cows. J. Am. Vet. Med. Assoc. 210:672-674. 
Thurmond M.C., Hietala S.K. \& Blanchard P.C. 1997b. Herd-Based diagnosis of Neospora caninum-induced endemic and epidemic abortion in cows and evidence for congenital and postnatal transmission. J. Vet. Diagn. Invest. 9:44-49.

Thrusfield M. 2007. Veterinary Epidemiology. Butterworths, London. 610p.

Tiwari A., VanLeeuwen J.A., Dohoo I.R., Keefe G.P., Haddad J.P., Trem-blay R., Scott H.M. \& Whiting T. 2007. Production effects of pathogens causing bovine leukosis, bovine viral diarrhea, paratuberculosis, and neosporosis. J. Dairy Sci. 90:659-669.

Trees A.J., Davison H.C., Innes E.A. \& Wastling J.M. 1999. Towards evaluating the economic impact of bovine neosporosis. Int. J. Parasitol. 29:1195-1200.

Williams D.J.L., Hartley C.S., Björkman C. \& Trees A.J. 2009. Endogenous and exogenous transplacental transmission of Neospora caninum: How the route of transmission impacts on epidemiology and control of disease. Parasitology 136, 1895-1900. 